\title{
Validity of routine biochemical and ultrasound scores for prediction of hepatic fibrosis and steatosis in NAFLD
}

\author{
Rasha Eletreby ${ }^{1 *}$ (D) Zeinab Abdellatif ${ }^{1}$, Yasmine Gaber ${ }^{1}$, Ahmed Ramadan', Naglaa Ahmad², Hany Khattab ${ }^{3}$, \\ Mohamed Said ${ }^{1}$ and Yasmin Saad ${ }^{1}$
}

\begin{abstract}
Background: We evaluated the validity of some non-invasive scores and ultrasound findings to predict fibrosis and steatosis in a cohort of NAFLD patients who underwent liver biopsy. Ninety-seven NAFLD patients were enrolled and classified into NASH (66) and simple steatosis groups (31) based on liver biopsy. ROC curves were constructed for Fibrosis-4 index (FIB4), aspartate aminotransferase to platelet ratio index (APRI), and NAFLD fibrosis score (NFS) in fibrosis prediction, also for (hepatic steatosis index; HSI, fatty liver index; FLI) and ultrasonographic subcutaneous and visceral adipose tissue measurements (SAT and VAT) for steatosis prediction.

Results: FIB4 had AUC of 0.6, APRI and NFS at cutoffs of 0.3 and -.2.4 had AUC of 0.64 and 0.63 in detecting the presence of any grade of fibrosis, and of $(0.52,0.55$, and 0.58$)$ for significant fibrosis. FIB4 at a cut-off of (0.76) had the highest AUC in detecting any grade of fibrosis in the simple steatosis group (0.81). SAT (at cutoff of 2.1 and 2.5) was superior to VAT. HSI (at cutoff 45.35 and 45.7) was superior to FLI in detecting moderate or marked steatosis.

Conclusion: FIB4 and NFS can be used in screening for silent liver disease with ongoing fibrosis in simple steatosis. They are unsatisfactory predictors for significant fibrosis in NAFLD. SAT is better than VAT in predicting moderate steatosis and is slightly better than biochemical HSI.
\end{abstract}

Keywords: NAFLD, Fibrosis progression, Steatosis, Non-invasive assessment

\section{Background}

NAFLD represents the most common liver disorder in Western countries, with $17-46 \%$ prevalence among adults [1]. In Egypt, the prevalence of NAFLD is rising owing to rising prevalence of obesity. NAFLD was found in $57.65 \%$ of a cohort of obese Egyptian adolescents in one study [2].

NAFLD is tightly associated with several risk factors; the presence of which impacts the severity and progression of the disease. The most important risk factors are known to be insulin resistance (IR) and metabolic syndrome (MetS) [3].

\footnotetext{
*Correspondence: r_m_tawfik@yahoo.com

'Endemic Medicine and Hepatology Department, Cairo University, Cairo, Egypt

Full list of author information is available at the end of the article
}

The diagnosis of NASH provides important prognostic information and indicates an increased risk of fibrosis progression, cirrhosis, and possibly HCC. It also prompts closer follow-up and possibly a greater need for more intensive therapy [4]. Similarly, steatosis should be documented whenever NAFLD is suspected as the primary disease or as a coexisting condition as it predicts future diabetes mellitus, cardiovascular events, and arterial hypertension [4].

Unfortunately, the standard diagnostic tool for NASH, namely, liver biopsy, has significant limitations such as sampling variability [5], being prohibitively expensive and relatively invasive with some morbidity and very rare mortality risk [6]. Also, quantification of fat content is not of interest in clinical practice, except as a surrogate of treatment efficacy, and is therefore not generally 
recommended. Imaging, especially ultrasound is the preferred tool for diagnosis of NAFLD being cheaper and more available than MRI; the gold standard. However, imaging has the limitation of low sensitivity in the detection of mild degrees of steatosis; hence, it is not suitable for screening purposes [4].

Recent guidelines started to support the use of noninvasive biomarkers and scores of fibrosis and steatosis, as well as transient elastography \pm controlled attenuation parameter (CAP), as acceptable non-invasive procedures for fibrosis/steatosis assessment in NAFLD cases [4].

Among non-invasive markers for fibrosis assessment, the FIB4 index has been independently validated in subjects with $\mathrm{HCV}$ infection. It is a simple and relatively inexpensive method that correlates with the stage of fibrosis [7]. Another simple fibrosis scoring system is the NAFLD fibrosis score (NFS) which was found to independently identify patients with and without advanced fibrosis at initial NAFLD diagnosis [8].

Fatty liver index (FLI) and hepatic steatosis index (HSI), on the other side; are steatosis scores that were found to reliably predict the presence, rather than the severity, of steatosis [9].

In the current study, we aim to evaluate the validity of three non-invasive fibrosis markers, namely, FIB-4, APRI, and NFS, in detecting both significant fibrosis in patients with NAFLD, and any stage of fibrosis in the subgroup of patients with simple steatosis. As well as to compare the diagnostic performance of two other noninvasive hepatic steatosis indices (HSI and FLI) to US findings (grading of liver brightness and subcutaneous and visceral fat measurements) versus liver biopsy as a gold standard, being cheap, simple, readily available indices for steatosis detection in NAFLD patients in the setting of low socioeconomic status and high prevalence, and morbidity of NAFLD.

\section{Methods}

The current study included 97 NAFLD patients who sought medical advice at NASH multi-disciplinary clinic, Kasr Alainy Hospital, Cairo University, Cairo, Egypt. The clinic involves a multidisciplinary team including hepatologists, nutritionists, pathologists, endocrinologists, endoscopists, and bariatric surgeons.

Among 250 patients presented to the NAFLD clinic, 97 patients enrolled after exclusion of those with positive viral hepatitis markers and those who did not consent for liver biopsy. The study was conducted in the period from July 2014 to July 2016.

Patients who fulfilled the following inclusion criteria were recruited.

- Age $\geq 18$ years.

- Bright liver by ultrasound.
- Negative hepatitis markers (negative HBsAg, $\mathrm{HBcAb}, \mathrm{HCVAb}$ ).

- Negative history of other chronic liver diseases as autoimmune hepatitis, primary biliary cirrhosis, etc.

Enrolled patients were subjected to the following:

- Informed consent: All patients enrolled had signed an informed consent form. The study was carried out per the Helsinki Declaration [10] and was approved by the ethical committee of the endemic medicine department, Kasr Alainy Hospital, Cairo University.

- Clinical evaluation including sex, age, body-mass index (BMI), history of DM, HTN, or any comorbidities, detailed dietary questionnaire, and anthropometric measurements.

- Laboratory investigations in the form of liver biochemical profile (BIL, AST, ALT, ALP, GGT, INR), lipid profile (cholesterol, triglycerides, HDL, LDL), and fasting blood glucose.

- Calculation of fibrosis indices:

- Calculation of APRI by the formula [(AST/upper limit of normal $\times 100) /$ platelet count] [11]. Where, non- significant fibrosis $(<\mathrm{F} 2):<0.7$, significant fibrosis ( $\geq \mathrm{F} 2-<\mathrm{F} 4)$ : $0.7-<1$ and cirrhosis (F4): $\geq 1$.

- Calculation of FIB-4 by the formula (Age $\times$ AST/ platelet count $\times$ sqr ALT) [12]. Non-significant fibrosis $(<$ F2) was identified as FIB4 $<1.45$, significant fibrosis ( $\geq$ F2 $-<F 4): 1.45-<3.25$ and cirrhosis (F4): $\geq 3.25$.

- Calculation of NAFLD fibrosis score:

The NFS is composed of 6 variables, including age, hyperglycemia, BMI, platelet count, albumin, and AST/ ALT ratio.

NAFLD fibrosis score $=-1.675+0.037 \times$ age $($ year $)+$ $0.094 \times$ BMI $(\mathrm{kg} / \mathrm{m} 2)+1.13 \times$ IFG/diabetes $($ yes $=1$, no $=0)+0.99 \times \mathrm{AST} / \mathrm{ALT}$ ratio $-0.013 \times$ platelet count $(\times$ $109 / \mathrm{L})-0.66 \times$ albumin $(\mathrm{g} / \mathrm{dL})$ [8]. NAFLD patients with a score less than -1.5 were classified as "low probability of advanced liver fibrosis," and those patients with a score of at least -1.5 were classified as "intermediate or high probability of advanced liver fibrosis" [8].

- Calculation of steatosis indices:

- Hepatic steatosis index (HSI) [13]

$\mathrm{HSI}=8 \times \mathrm{ALT} / \mathrm{AST}+\mathrm{BMI}+2$, if $\mathrm{DM} ;+2$, if female with values $<30$ ruling out and values $>36$ ruling in steatosis.

- Fatty liver index (FLI) [14]

FLI $=\operatorname{logistic}(0.953 \times \ln (\mathrm{TG})+0.139 \times \mathrm{BMI}+0.718 \times$ $\ln (\gamma \mathrm{GT})+0.053 \times$ waist-15.745) $\times 100$; where $\operatorname{logistic}(\mathrm{x})$ 
$=1 /\left(1+\mathrm{e}^{-\mathrm{x}}\right)$ denotes the logistic function and ln the natural logarithm.

Values $<30$ rule out and values $>60$ rules in steatosis.

- Abdominal ultrasonography: Real-time abdominal US was done using a transabdominal 4C-AH46701AA machine with a 1-5MHZ convex linear transducer, with a built-in color flow mapping (CFM) and Doppler functions. US evaluation included subcutaneous adipose tissue (SAT) measurement and visceral adipose tissue (VAT) measurement. The appearance of the liver concerning size, echo pattern, presence of hepatic focal lesion/s and portal vein patency, scanning for spleen, examination for ascites, abdominal lymphadenopathy, and abdominal masses, together with scanning for other abdominal and pelvic organ.

- Liver biopsy: All enrolled patients had undergone liver biopsy to differentiate NAFL from NASH, with the calculation of NAFLD activity score (NAS). The NAS is the sum of the biopsy scores for steatosis (0 to 3), lobular inflammation (0 to 3), and hepatocellular ballooning (0 to 2), in addition to the calculation of the fibrosis stage (0 to 4$)$. A NAS $<3$ corresponds to NAFL, 3 to 4 corresponds to borderline NASH, and a score $\geq 5$ corresponds to NASH [15]. Steatosis grades were also determined (mild, moderate, severe) [16].

\section{Statistical methods}

- Numerical variables are presented as mean (standard deviation); while categorical variables are presented as numbers and percentages.

- Logistic regression analysis was done to identify predictors of the presence of NASH, NAS score $\geq 5$, and to identify predictors of NAS score $>4$. Logistic regression analysis was done to identify factors associated with higher grades of fibrosis.

- ROC curves were constructed to analyze the discriminatory power of non-invasive fibrosis markers, Fib4, APRI, and NFS, for the prediction of the presence of fibrosis, and another time for predicting significant fibrosis, with liver biopsy being the gold standard.

- (ROC) curve analysis was used to identify the best cut-off value of various liver fat indices for detecting moderate/marked steatosis

- Data analysis was done using Statistics/Data Analysis (STATA) version 13.1 software.

\section{Results}

\section{Baseline characters of the study population}

Mean age was 42 years, $74.2 \%$ were females, mean BMI was $34.3,20.6 \%$ were diabetic, $59 \%$ had macro- and microvesicular steatosis in liver biopsy. 17/80 (21.3\%) patients had severe steatosis. In total, 20.6\% (20/97) had significant fibrosis ( $\geq$ F2). Two patients (2.1\%) had severe inflammation. Two subgroups have been then identified: the NASH group (66 patients) and the simple steatosis group (31 patients). DM, female waist circumference, fasting blood sugar, and fibrosis stage were statistically different between both groups (Table 1). Patients with marked steatosis had statistically significant higher ALT ( $p$ 0.04), as well as a higher number of patients with grade III liver brightness by ultrasound $(p<0.0001)$.

\section{Risk factors for significant and higher grades of fibrosis} Albumin, bilirubin, and NFS were significantly associated with significant fibrosis on performing univariate logistic regression (Table 2).

\section{Risk factors for the presence of fibrosis in simple steatosis subgroup}

Higher age, lower serum albumin, NFS, and FIB4 were significantly associated with the presence of fibrosis despite the absence of inflammation in studied biopsies (Table 3).

\section{Sensitivity and specificity of different indices for fibrosis prediction}

At a cutoff of 0.76 , FIB4 was $83.3 \%$ sensitive, $69.2 \%$ specific, with an accuracy of $77.4 \%$, positive likelihood ratio $(\mathrm{LR}+)$ of 2.7, and negative likelihood ratio (LR-) of 0.2 for detection of fibrosis in patients with simple steatosis. APRI had sensitivity, specificity, and accuracy rates of $66.1 \%, 61.3 \%$, and $65.6 \%$ at the cutoff of 0.30 in the detection of any grade of fibrosis. A cutoff value of -2.44 NFS had sensitivity, specificity, LR+, and LR- of $73.44 \%$, $51.6 \%,+1.52$, and -0.51 in detecting the presence of any grade of fibrosis (Fig. 1).

\section{AUC of different markers in fibrosis prediction}

FIB4, APRI, and NFS had acceptable and comparable AUC of 0.60, 0.64, 0.63 in detecting the presence of any grade of fibrosis, however, all markers were poor predictors of significant fibrosis $\geq$ F2 (AUC: $0.52,0.55,0.58$ ) (Fig. 1, Table 4).

In the subgroup of patients with simple steatosis (NAS score of $\leq 4$ ); FIB4 had the highest AUC curve compared to NFS and APRI in the detection of any grade of fibrosis ( 0.81 versus 0.74 and 0.78 ) (Fig. 1 , Table 4).

\section{AUC for HSI and FLI in the prediction of different grades of steatosis}

HSI was superior to FLI in detecting either moderate or marked steatosis. At the cutoff of 45.35 , HSI had the sensitivity of $73.17 \%$, specificity of $58.06 \%$, LR+ of 1.74 , LR- of 0.5 , and accuracy of $66.67 \%$ in predicting moderate steatosis with AUC of 0.64 , and nearly the same percentages with less accuracy in detecting marked steatosis 
Table 1 Baseline characteristics of the study population

\begin{tabular}{|c|c|c|c|c|c|}
\hline & & The whole group & NAS $<4(n=31)$ & $\begin{array}{l}\text { NAS } \geq 4 \\
(n=66)\end{array}$ & $P$ value \\
\hline \multicolumn{2}{|l|}{ Age } & $42.02(8.99)$ & $42(10.44)$ & $42.03(8.30)$ & 0.9 \\
\hline \multicolumn{2}{|l|}{$\begin{array}{l}\text { Gender } \\
\text { Male/female }\end{array}$} & $25(25.8 \%) / 72(74.2 \%)$ & $10(32.2 \%) / 21(67.7 \%)$ & $15(22.72 \%) / 51$ (77.27\%) & 0.3 \\
\hline \multicolumn{2}{|l|}{$\begin{array}{l}\text { Residence } \\
\text { Rural/urban }\end{array}$} & $18(27.3 \%) / 48$ (72.7\%) & $4(12.9 \%) / 16(51.61 \%)$ & $14(21.21 \%) / 32(48.48 \%)$ & 0.4 \\
\hline \multicolumn{2}{|l|}{ Diabetes mellitus } & $20(20.6 \%)$ & $2(6.45 \%)$ & $18(27.27 \%)$ & 0.01 \\
\hline \multicolumn{2}{|l|}{ Hypertension } & $13(13.40 \%)$ & $2(6.45 \%)$ & $11(16.67 \%)$ & 0.1 \\
\hline \multicolumn{2}{|l|}{ BMI median (IQR) } & $32.9(29.15-38.14)$ & $32(28-35.99)$ & $33.05(30-38.86)$ & 0.2 \\
\hline \multirow[t]{2}{*}{ Waist circumference } & $\begin{array}{l}\text { Males } \\
\text { Median (IQR) }\end{array}$ & $55(16.84 \%)$ & $44(40-50)$ & $52(49-62)$ & 0.07 \\
\hline & $\begin{array}{l}\text { Females } \\
\text { Median (IQR) }\end{array}$ & $81(38.41 \%)$ & $48.5(45-56)$ & $68(55-127)$ & 0.01 \\
\hline \multicolumn{6}{|c|}{ Laboratory parameters } \\
\hline \multicolumn{2}{|l|}{ Hemoglobin } & $12.91(1.70)$ & $12.61(2.08)$ & $13.05(1.49)$ & 0.2 \\
\hline \multicolumn{2}{|l|}{ Platelets } & $272.12(69.69)$ & $272.97(62.003)$ & $271.72(73.52)$ & 0.9 \\
\hline \multicolumn{2}{|l|}{ AST median (IQR) } & $30(22-41.5)$ & $27(22-34)$ & $30(23-44)$ & 0.06 \\
\hline \multicolumn{2}{|l|}{ ALT median (IQR) } & $32.5(21-57)$ & $26(18-38)$ & $40(23-60)$ & 0.01 \\
\hline \multicolumn{2}{|c|}{ Bilirubin median (IQR) } & $0.56(0.4-0.9)$ & $0.5(0.4-0.84)$ & $0.6(0.4-0.9)$ & 0.8 \\
\hline \multicolumn{2}{|l|}{ Albumin } & $4.27(0.43)$ & $4.21(0.48)$ & $4.30(0.41)$ & 0.4 \\
\hline \multicolumn{2}{|l|}{ INR } & $1.001(0.09)$ & $1.01(0.05)$ & $0.99(0.10)$ & 0.7 \\
\hline \multicolumn{2}{|c|}{ Cholesterol median (IQR) } & $202(177-229)$ & $202(166-225)$ & $202(180-234)$ & 0.4 \\
\hline \multicolumn{2}{|c|}{ Triglycerides median (IQR) } & $140(100-180)$ & $141(96-200)$ & $135.5(100-179.5)$ & 0.9 \\
\hline \multicolumn{2}{|l|}{ FBG median (IQR) } & $99.5(90-110)$ & $92(86-101)$ & $100(90-116)$ & 0.04 \\
\hline \multicolumn{6}{|l|}{ Liver biopsy } \\
\hline \multirow[t]{3}{*}{ Type of steatosis } & Macro and microvesicular & 29 (59.2\%) & 10 & 19 & 0.3 \\
\hline & Macrovesicular & $12(24.5 \%)$ & 4 & 8 & \\
\hline & Microvesicular & $8(16.3 \%)$ & 5 & 3 & \\
\hline \multirow[t]{5}{*}{ Fibrosis stage } & Fo & 31 (31.96\%) & 13 & 18 & 0.04 \\
\hline & F1 & $46(47.42 \%)$ & 17 & 29 & \\
\hline & $\mathrm{F} 2$ & $17(17.5 \%)$ & 1 & 16 & \\
\hline & F3 & $2(2.1 \%)$ & 0 & 2 & \\
\hline & F4 & $1(1.03 \%)$ & 0 & 1 & \\
\hline
\end{tabular}

Unless otherwise stated, numerical variables presented as mean (SD)

at a cutoff value of 45.7 (sensitivity was $73.33 \%$, specificity was $50.88 \%$, LR+ was 1.49 , LR- was 0.52 , while accuracy was 55.6\%) with AUC of 0.66 . On the other hand, FLI had AUCs of 0.52 and 0.56 for predicting the presence of moderate and marked steatosis (Fig. 2).

\section{AUC for ultrasound parameters (SAT and VAT) in the prediction of different grades of steatosis}

SAT shows a higher AUC for predicting moderate steatosis (AUC 0.66 vs 0.56) and nearly equal AUC as VAT for detecting marked steatosis (AUC 0.57 vs 0.60). A cutoff value of 2.1, SAT had a sensitivity of $81.25 \%$, specificity of $60 \%$, LR+ of 2.03 , LR- of 0.3 , and accuracy of $73.08 \%$ in detecting moderate steatosis. For predicting marked steatosis, SAT had a sensitivity of $62.5 \%$, specificity of $66.7 \%$, LR+ of 1.9, LR- of 0.6 , and accuracy of $65.4 \%$ at cutoff value of 2.5 . On the other hand, at a cutoff value of 1.8, VAT showed a sensitivity of $66.67 \%$, specificity of $52.63 \%$, LR+ of $1.41, \mathrm{LR}-$ of 0.63 , and accuracy of $58.06 \%$ in predicting marked steatosis (Fig. 3).

\section{Discussion}

In this cross-sectional observational study, we aimed to evaluate the role of some non-invasive, simple, readily 
Table 2 Univariate logistic regression analysis for factors associated with significant fibrosis $\geq$ F2 in NAFLD patients

\begin{tabular}{lll}
\hline & Odds ratio (95\% conf. interval) & $\boldsymbol{P}$ value \\
\hline Age & $1.01(0.96-1.06)$ & 0.7 \\
Gender (female) & $1.62(0.62-4.18)$ & 0.3 \\
DM & $3.24(0.9-12.03)$ & 0.07 \\
BMI > 35 & $1.18(0.49-2.87)$ & 0.7 \\
AST & $1.02(0.99-1.05)$ & 0.1 \\
ALT & $1.004(0.99-1.02)$ & 0.5 \\
ALP & $1.01(0.99-1.02)$ & 0.3 \\
GGT & $0.99(0.98-1.004)$ & 0.2 \\
Triglycerides & $0.99(0.99-1.001)$ & 0.1 \\
Cholesterol & $0.99(0.98-1.01)$ & 0.4 \\
Albumin & $0.31(0.11-0.89)$ & $\mathbf{0 . 0 3}$ \\
Bilirubin & $7.19(1.59-32.50)$ & $\mathbf{0 . 0 1}$ \\
APRI & $3.18(0.52-19.51)$ & 0.2 \\
FIB4 & $2.17(0.80-5.87)$ & 0.1 \\
NFS & $1.41(1.1-1.89)$ & $\mathbf{0 . 0 2}$ \\
\hline
\end{tabular}

available, and cheap indices, namely, FIB-4, APRI, and NFS in the detection of significant fibrosis ( $\geq$ F2) in patients with NAFLD, as well as their role in detecting any stage of fibrosis in a subgroup of patients with simple steatosis. Similarly, we could evaluate the predictability of other non-invasive steatosis indices (HSI and FLI) and compare them to US findings (grading of liver brightness and subcutaneous and visceral fat measurements) taking liver biopsy results as a standard in diagnosis and grading of hepatic steatosis/fibrosis.

Significant fibrosis ( $\geq$ F2) was observed in $20.6 \%$, while advanced fibrosis (F3-4) was seen in $3.1 \%$ of patients.

Table 3 Predictors of higher grades of fibrosis in simple steatosis group (NAS score $\leq 4)(n=31)$

\begin{tabular}{lll}
\hline & Odds ratio (95\% conf. interval) & $\boldsymbol{P}$ value \\
\hline Age & $1.1(1.04-1.18)$ & 0.04 \\
Gender (female) & $3(0.63-14.23)$ & 0.1 \\
BMI > 35 & $1.12(0.24-5.21)$ & 0.9 \\
AST & $1.06(0.98-1.14)$ & 0.1 \\
ALT & $1.01(0.97-1.05)$ & 0.6 \\
ALP & $0.99(0.98-1.02)$ & 0.9 \\
GGT & $0.99(0.98-1.01)$ & 0.7 \\
Triglycerides & $0.99(0.98-1.003)$ & 0.2 \\
Cholesterol & $0.99(0.98-1.01)$ & 0.6 \\
Albumin & $0.1(0.01-0.67)$ & 0.02 \\
FIB4 & $10.73(1.41-81.54)$ & 0.02 \\
NFS & $1.94(1.11-3.40)$ & 0.02 \\
\hline
\end{tabular}

According to the National Health and Nutrition Examination Survey conducted in 1988-1994, advanced fibrosis (NFS > 0.676) was only observed in $3.2 \%$ of patients with NAFLD [17].

In this study, the prevalence of T2DM was significantly higher in the NASH group compared to the simple steatosis one. It is well known that diabetes risk and type 2 DM (T2DM) are closely associated with the severity of NAFLD, progression to NASH, advanced fibrosis, and HCC development [18].

Regression analysis for predictors of significant fibrosis revealed that lower serum albumin, higher bilirubin, and NFS were significantly associated with higher grades of fibrosis ( $\geq$ F1). Several studies revealed that NFS is an important predictor of advanced fibrosis in NAFLD patients [19].

Although patients with simple steatosis without inflammation were considered for a long time to have a benign course with little progression [1], this view has been modified by results of different studies demonstrating that steatosis alone may progress to NASH with fibrosis, however, at a slower rate [20].

In the subgroup of patients with simple steatosis, predictors of fibrosis were older age, lower serum albumin, FIB4, and NFS. The importance of identifying patients who had fibrosis is that this could carry the risk of progressing to NASH. In one study by Pais et al., $64 \%$ of 25 patients with simple steatosis developed NASH after an average of 3.7 years. They showed that severe ballooning, presence of bridging fibrosis, older age, and deterioration of metabolic risk factors were associated with a more rapid progression [21].

In the current study, FIB4, APRI, and NFS had comparable AUC for detecting the presence of any grade of fibrosis. Detecting the early stages of fibrosis is of great importance in preventing disease progression to minimize complications [22]. On the other hand, all markers were poor predictors of significant fibrosis $\geq$ F2. Contrarily, Castera in 2018 reported AUC for transient elastography, FIB-4, and the NAFLD fibrosis scores in diagnosing severe fibrosis-cirrhosis of $0.88,0.84$, and 0.84 respectively [23]. Mohamed et al. studied the diagnostic performance of FIB4, NFS, and APRI in NAFLD. They found AUC for advanced fibrosis of 0.936, 0.916, and 0.907 [24]. Also, in a meta-analysis of 13 studies consisting of 3064 patients, NFS had an AUROC of 0.85 for predicting advanced fibrosis [25]. This difference may be related to the small number of patients with advanced fibrosis $(\geq$ F3) in our study (3\%).On the other hand, Nones et al. evaluated results of FIB4, APRI, and NFS in 67 patients with NAFLD, the best diagnostic accuracy was achieved with FIB 4 model $($ AUROC $=0.83$ ) versus APRI and NFS. Again, the difference may be attributed to the higher number of patients with advanced 


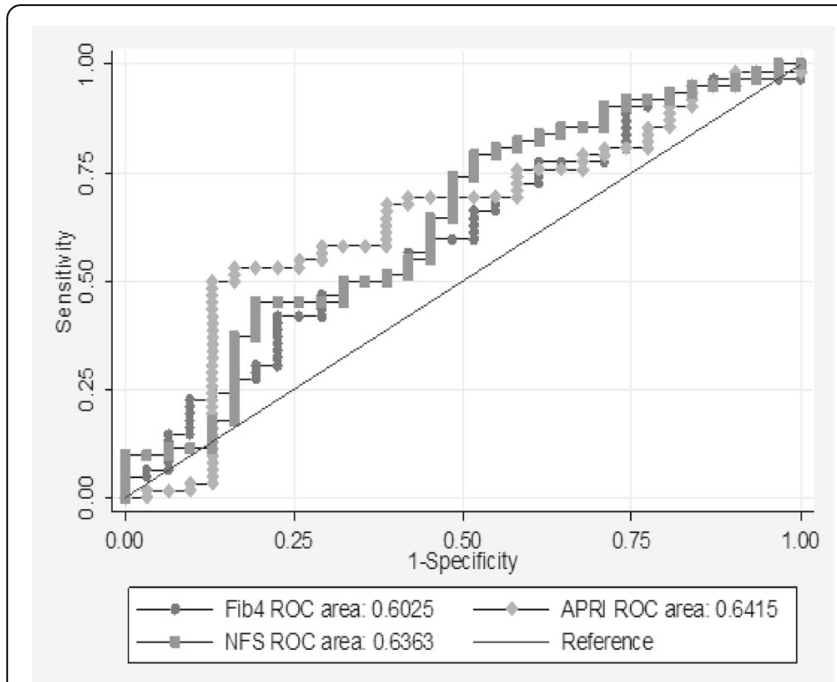

\section{A) Fib-4, APRI, NFS in predicting any degree of fibrosis in the whole study population}

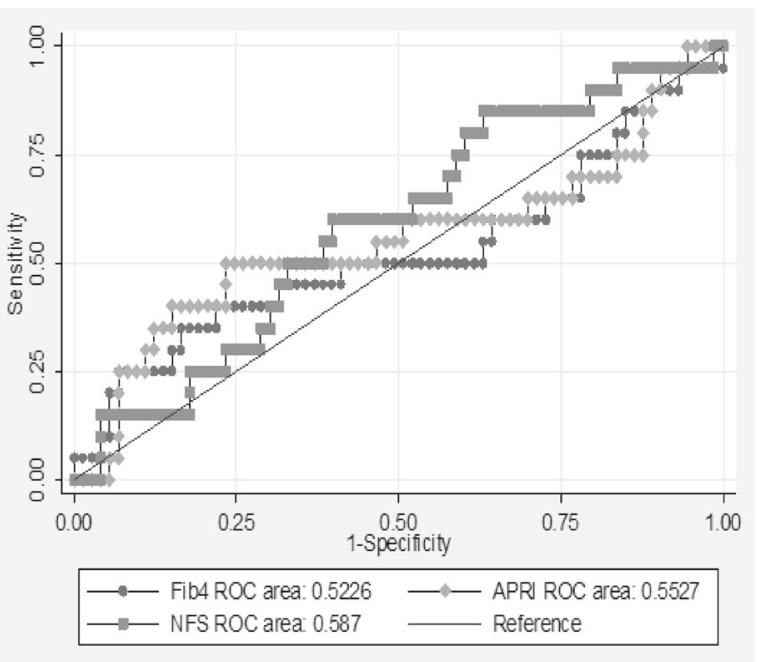

B) Fib-4, APRI, and NFS in predicting significant fibrosis ( $\geq F 2$ ) in the whole study population

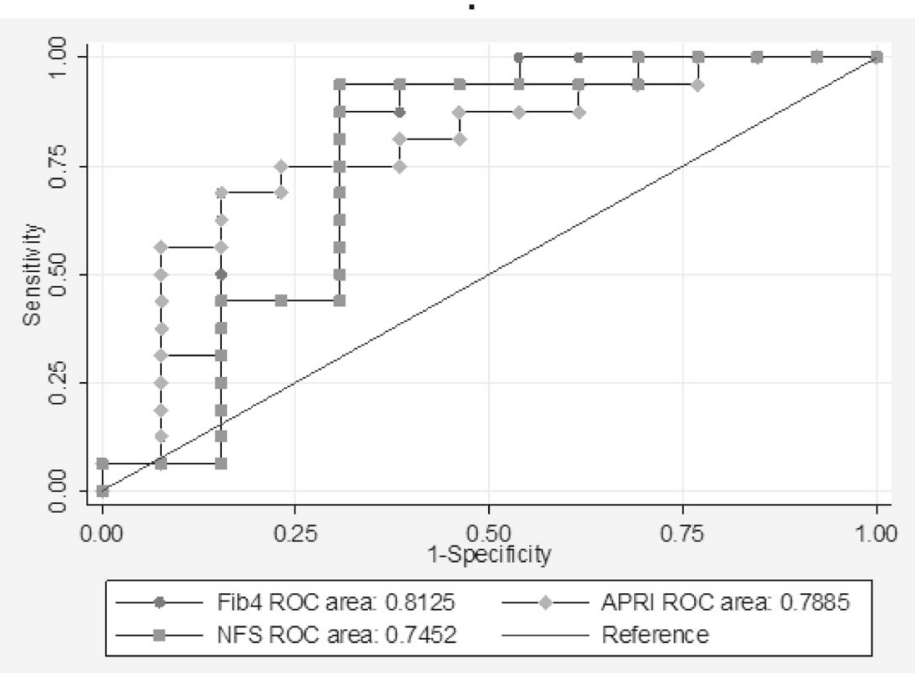

\section{C) Fib-4, APRI, NFS in predicting any degree of fibrosis in the subset of patients with simple steatosis (NAS $\leq 4)$}

Fig. 1 ROC curves for predictivity of FIB4, APRI, NFS of different degrees of fibrosis. (A) Fib-4, APRI, NFS in predicting any degree of fibrosis in the whole study population. (B) Fib-4, APRI, and NFS in predicting significant fibrosis ( $\geq$ F2) in the whole study population. (C) Fib-4, APRI, NFS in predicting any degree of fibrosis in the subset of patients with simple steatosis (NAS $\leq 4$ )

fibrosis ( $\geq$ F3) in his study $18(26.86 \%)$ compared to the current one (3\%) [26].

Among non-invasive scores of hepatic steatosis, the hepatic steatosis index (HSI) has been derived from data of a Korean cross-sectional study that involved more than 10,000 patients and has been validated against ultrasound [13]. It is a promising score that seems to predict incident metabolic syndrome [27]. In the current study, HSI has been validated against liver biopsy results and showed acceptable AUC in the prediction of moderate and marked steatosis. Our results go hand in hand with those of Fedchuk et al., where HSI had an AUC of 0.65 in the prediction of the presence of hepatic steatosis [9].

The fatty liver index (FLI) was mentioned as one of the best-validated steatosis scores, as it has been externally validated in the general population or grade 3 
Table 4 AUROC of tested markers in detection of presence of fibrosis as well as significant fibrosis in the whole cohort and in simple steatosis subgroup

\begin{tabular}{llll}
\hline & \multicolumn{2}{l}{ AUROC (95\% conf. interval) for detection of } \\
\cline { 2 - 4 } & Presence of fibrosis & Significant fibrosis & Presence of fibrosis in simple steatosis subgroup \\
\hline APRI & $\begin{array}{l}0.64(0.52-0.76) \\
\text { (Cutoff: } 0.30)\end{array}$ & $0.55(0.4-0.72)$ & $0.788(0.61-0.97)$ \\
FIB4 & $0.60(0.48-0.73)$ & $0.52(0.4-0.69)$ & $0.81(0.64-0.98)$ \\
NFS & $0.64(0.51-0.76)$ & $0.58(0.45-0.73)$ & $0.74(0.53-0.95)$ \\
\hline
\end{tabular}

obese persons and can variably predict metabolic, hepatic, and cardiovascular outcomes/mortality [4]. However, in the current study, FLI had unsatisfactory AUC for predicting the presence of moderate and marked steatosis and was inferior to HSI. The discrepancy maybe since the fatty liver index (FLI) has only been validated against liver ultrasonography in published studies [28] rather than liver biopsy that has been taken in our study.
Despite its limitations, ultrasound is still recommended by EASL 2016 guidelines to be the preferred first-line diagnostic procedure for imaging of NAFLD, as it provides additional diagnostic hepatobiliary information [4]. In the current study, subcutaneous adipose tissue (SAT) showed better AUC than visceral adipose tissue (VAT) in predicting moderate steatosis and similar AUC in predicting marked hepatic steatosis. Lower AUC for detection of marked steatosis in the current
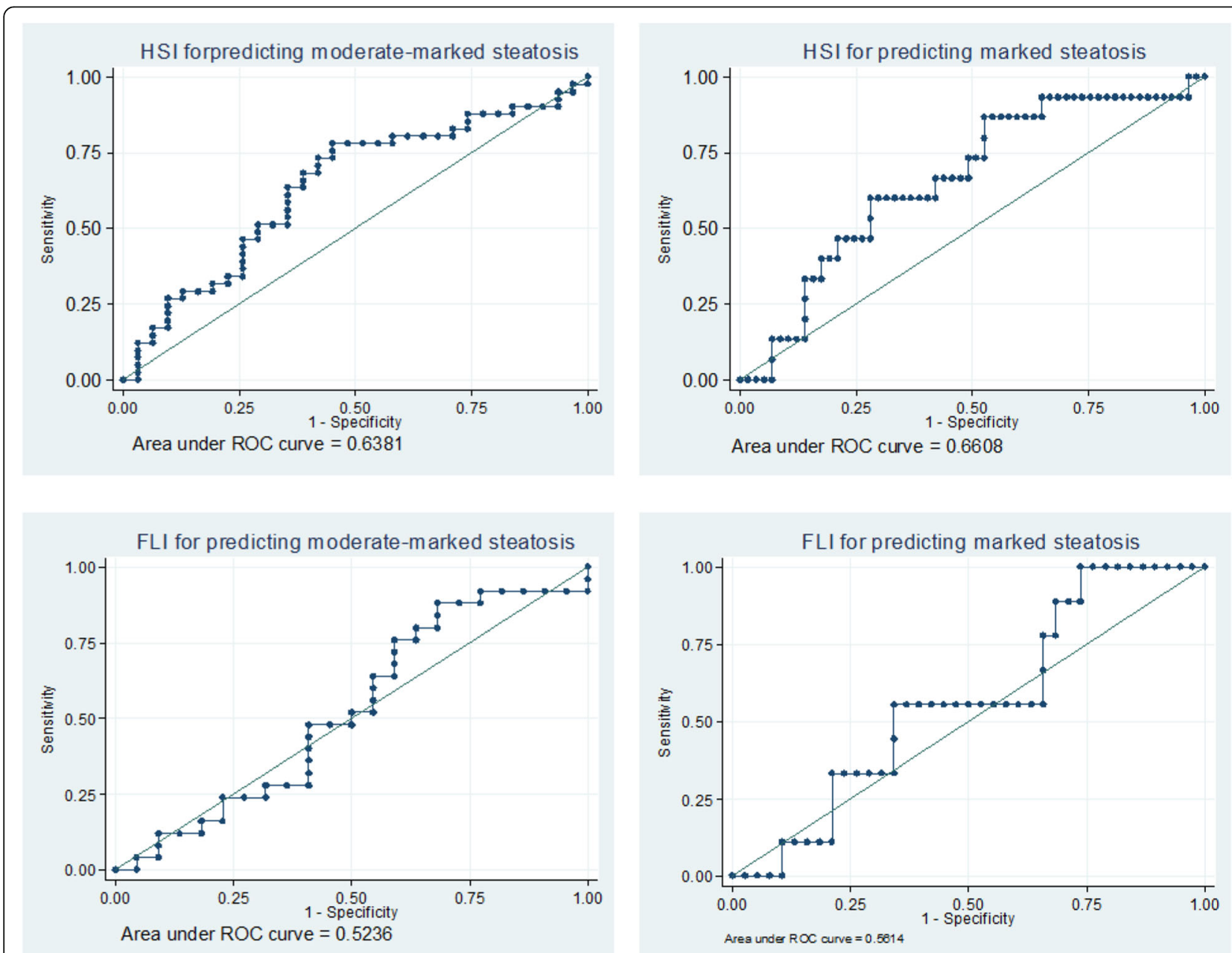

Fig. 2 ROC curve for $\mathrm{HSI}$ and FLI in the prediction of moderate/marked steatosis 


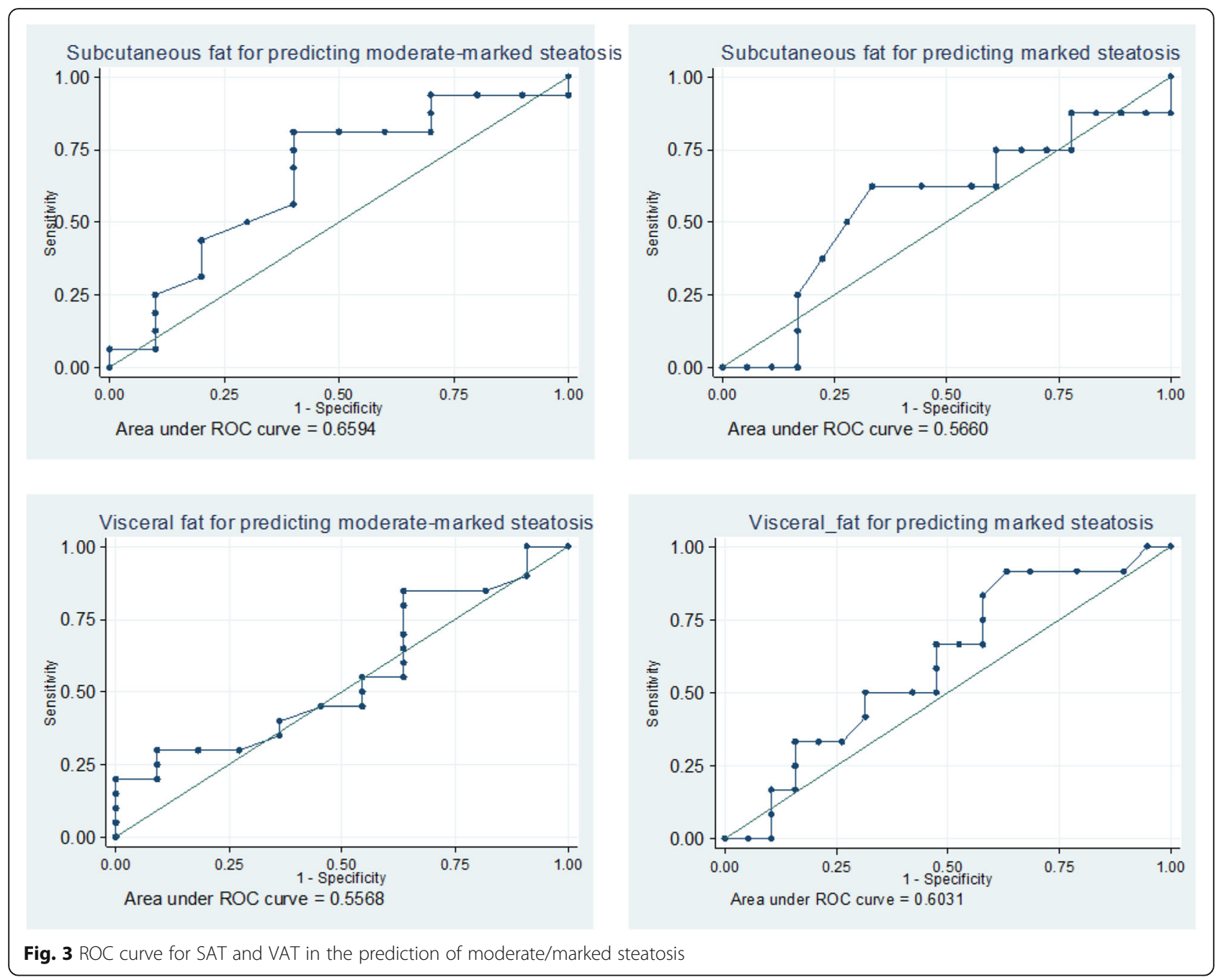

study may be due to the technical limitation of higher waist circumference and obesity of this cohort.

In the current study, subcutaneous adipose tissue (SAT) showed the best results among all tested noninvasive measures, namely, serum biomarkers (HSI and FLI) as well as visceral adipose tissue (VAT) by ultrasound in detecting moderate steatosis.

The main point of strength of the current study is the presence of liver biopsy results as a gold standard for comparison with non-invasive markers. Note that many other published studies lack the results of liver biopsy and compare the accuracy of non-invasive biochemical markers with imaging or controlled attenuation parameter (CAP) measures which are still not considered the gold standard for fibrosis/steatosis diagnosis. The main limitation of our study is that the main cohort of patients has no or mild degree fibrosis (F0-F1) by liver biopsy (79\%), while significant and advanced fibrosis was present in $21 \%$ which may have affected the results.

\section{Conclusion}

Based on the results of the current study, we can conclude that FIB4 could be used as a screening tool for silent liver disease with ongoing fibrosis in NAFL D patients with simple steatosis, which warrants closer follow-up and more intensive therapies. However, FIB4, APRI, and NFS are still unsatisfactory non-invasive markers for the detection of significant fibrosis in NASH patients and further studies are needed to reach optimal markers. We can also conclude that ultrasound assessment of subcutaneous adipose tissue (SAT) rather than visceral adipose tissue (VAT) performs well in the prediction of moderate steatosis and slightly better than hepatic steatosis index (HSI) which proves also to be a good noninvasive biochemical tool in the prediction of moderate steatosis and performs better than the fatty liver index. 


\section{Abbreviations}

APRI: AST to platelet ratio index; FIB 4: Fibrosis score 4; NAFLD: Non-alcoholic fatty liver disease; NAS: NAFLD activity score; NASH: Non-alcoholic steatohepatitis; NFS: NAFLD fibrosis score

\section{Acknowledgements}

No agency to be acknowledged

\section{Authors' contributions}

The authors read and approved the final manuscript; all authors contributed to the work. MS: Idea of the study. Revision of the manuscript. YS: Idea of the study, revision of the manuscript, and mastering the clinical work and connections with other specialists. RE: Drafting the manuscript and the corresponding author. ZA: Doing the statistical tests for the study. AR: Patient follow-up in the clinic and collecting data. YG: Patient follow-up in the clinic and collecting data. NA: Clinical follow-up of patients from the endocrinological point of view. HK: Doing the histopathological examination for liver biopsies. Taken from patients for the staging of steatosis and fibrosis.

\section{Funding}

No funding agent

\section{Availability of data and materials}

Data are available.

\section{Declarations}

\section{Ethics approval and consent to participate}

All patients signed informed consent. The study was carried out following the Helsinki Declaration [10] and was approved by the local ethical committee of the Endemic Medicine Department, Kasr Alainy Hospital, Cairo University; hence, it did not have an ethics committee's reference number.

\section{Consent for publication}

Patients had signed informed consent for use of their data in research and publication without the appearance of their names.

\section{Competing interests}

No competing interests

\section{Author details}

${ }^{1}$ Endemic Medicine and Hepatology Department, Cairo University, Cairo, Egypt. ${ }^{2}$ Internal Medicine Department, Endocrine Section, Faculty of Medicine, Cairo University, Cairo, Egypt. ${ }^{3}$ Pathology Department, Faculty of Medicine, Cairo University, Cairo, Egypt.

\section{Received: 11 January 2021 Accepted: 20 May 2021}

Published online: 31 May 2021

\section{References}

1. Vernon G, Baranova A, Younossi ZM (2011) Systematic review: the epidemiology and natural history of non-alcoholic fatty liver disease and non-alcoholic steatohepatitis in adults. Aliment Pharmacol Ther 34(3):274285. https://doi.org/10.1111/j.1365-2036.2011.04724.x

2. Zaki ME, Ezzat W, Elhosary YA, Saleh OM (2013) Factors associated with nonalcoholic fatty liver disease in obese adolescents Macedonian. J Med Sci 6:273-277

3. Gaggini M, Morelli M, Buzzigoli E, DeFronzo RA, Bugianesi E, Gastaldelli A (2013) Non-alcoholic fatty liver disease (NAFLD) and its connection with insulin resistance, dyslipidemia, atherosclerosis, and coronary heart disease. Nutrients 5(5):1544-1560. https://doi.org/10.3390/nu5051544

4. (2016) EASL-EASD-EASO Clinical Practice Guidelines for the management of non-alcoholic fatty liver disease. J Hepatol 64(6):1388-1402. https://doi. org/10.1016/j.jhep.2015.11.004

5. Ratziu V, Charlotte F, Heurtier A, Gombert S, Giral P, Bruckert E, Grimaldi A, Capron F, Poynard T, LIDO Study Group (2005) Sampling variability of liver biopsy in nonalcoholic fatty liver disease. Gastroenterology 128(7):18981906. https://doi.org/10.1053/j.gastro.2005.03.084

6. Chalasani N, Younossi Z, Lavine JE, Charlton M, Cusi K, Rinella M et al (2018) The diagnosis and management of nonalcoholic fatty liver disease practice guidance from the American Association for the Study of Liver Diseases. Hepatology 67(1)

7. Shah AG, Lydecker A, Murray K, Tetri BN, Contos MJ, Sanyal AJ, NASH Clinical Research Network (2009) Use of the FIB4 index for non-invasive evaluation of fibrosis in non-alcoholic fatty liver disease. Clin Gastroentero Hepatol 7(10):1104-1112. https://doi.org/10.1016/j.cgh.2009.05.033

8. Angulo P, Hui JM, Marchesini G, Bugianesi E, George J, Farrell GC, Enders F, Saksena S, Burt AD, Bida JP, Lindor K, Sanderson SO, Lenzi M, Adams LA, Kench J, Therneau TM, Day CP (2007) The NAFLD fibrosis score: a noninvasive system that identifies liver fibrosis in patients with NAFLD. Hepatology 45(4):846-854. https://doi.org/10.1002/hep.21496

9. Fedchuk L, Nascimbeni F, Pais R, Charlotte F, Housset C, Ratziu V, the LIDO Study Group (2014) Performance and limitations of steatosis biomarkers in patients with nonalcoholic fatty liver disease. Aliment Pharmacol Ther 40(10):1209-1222. https://doi.org/10.1111/apt.12963

10. World Medical Association (2013) Declaration of Helsinki: ethical principles for medical research involving human subjects. JAMA 310:2191-2194

11. Shaheen AA, Myers RP (2007) Diagnostic accuracy of the aspartate aminotransferase-to-platelet ratio index for the prediction of hepatitis Crelated fibrosis: a systematic review. Hepatology 46(3):912-921. https://doi. org/10.1002/hep.21835

12. Sterling RK, Lissen E, Clumeck N, Sola R, Correa MC, Montaner J (2006) et. al: Development of a simple noninvasive index to predict significant fibrosis patients with HIV/HCV co-infection. Hepatology 43(6):1317-1325. https://doi. org/10.1002/hep.21178

13. Lee $\mathrm{JH}$, Kim D, Kim HJ, Lee CH, Yang Jl, Kim W, Kim YJ, Yoon JH, Cho SH, Sung MW, Lee HS (2010) Hepatic steatosis index: a simple screening tool reflecting nonalcoholic fatty liver disease. Dig Liver Dis 42(7):503-508. https://doi.org/10.1016/j.dld.2009.08.002

14. Bedogni G, Bellentani S, Miglioli L, Masutti F, Passalacqua M, Castiglione A Tiribelli C (2006) The fatty liver index: a simple and accurate predictor of hepatic steatosis in the general population. BMC Gastroenterol 6(1):33. https://doi.org/10.1186/1471-230X-6-33

15. Kleiner DE, Brunt EM, Van Natta M, Behling C, Contos MJ, Cummings OW et al (2005) Design and validation of a histological scoring system for nonalcoholic fatty liver disease. Hepatology. 41(6):1313-1321. https://doi. org/10.1002/hep.20701

16. Brunt EM, Janney CG, Di Bisceglie AM, Neuschwander-Tetri BA, Bacon BR (1999) Nonalcoholic steatohepatitis: a proposal for grading and staging the histological lesions. Am J Gastroenterol 94(9):2467-2474. https://doi.org/1 0.1111/j.1572-0241.1999.01377.x

17. Kim D, Kim WR, Kim HJ, Therneau TM (2013) Association between noninvasive fibrosis markers and mortality among adults with nonalcoholic fatty liver disease in the United States. Hepatology. 57(4):1357-1365. https:// doi.org/10.1002/hep.26156

18. Loomba R, Abraham M, Unal A, Wilson L, Lavine J, Doo E et al (2012) Association between diabetes, family history of diabetes, and risk of nonalcoholic steatohepatitis and fibrosis. Hepatology 56(3):943-951. https:// doi.org/10.1002/hep.25772

19. Pérez-Gutiérrez OZ, Hernández-Rocha C, Candia-Balboa RA, , Arrese MA, Benítez C, Brizuela-Alcántara DC, Méndez-Sánchez N, Uribe M, Chávez-Tapia NC Validation study of systems for noninvasive diagnosis of fibrosis in nonalcoholic fatty liver disease in Latin population. Ann Hepatol 2013; 12: 416-424, 3, DOI: https://doi.org/10.1016/S1665-2681(19)31004-X.

20. Harrison SA, Torgerson S, Hayashi PH (2003) The natural history of nonalcoholic fatty liver disease: a clinical histopathological study. Am J Gastroenterol 98(9):2042-2047. https://doi.org/10.1111/j.1572-0241.2003. 07659.x

21. Pais R, Charlotte F, Fedchuk L, Bedossa P, Lebray P, Poynard T, Ratziu V, LIDO Study Group (2013) A systematic review of follow-up biopsies reveals disease progression in patients with non-alcoholic fatty liver. J Hepatol 59(3):550-556. https://doi.org/10.1016/j.jhep.2013.04.027

22. Cleveland E, Bandy A, Van-Wagner LB (2018) Diagnostic challenges of nonalcoholic fatty liver disease/nonalcoholic steatohepatitis. Clin Liver Dis $11(4)$

23. Castera (2018) Diagnosis of non-alcoholic fatty liver disease/non-alcoholic steatohepatitis: non-invasive tests are enough. Liver Int 38:67-70

24. Mohamed RA, Nabiha MI, ElShobakya MB, Khattab HM (2014) The value of noninvasive scoring systems for the diagnosis of advanced fibrosis in Egyptian patients with nonalcoholic fatty liver disease. Egypt Soc Intern Med 26(4):162-169. https://doi.org/10.4103/1110-7782.148151 
25. Musso G, Gambino R, Cassader M, Pagano G (2011) Meta-analysis: Natural history of non-alcoholic fatty liver disease (NAFLD) and diagnostic accuracy of non-invasive tests for liver disease severity. Ann Med 43(8):617-649. https://doi.org/10.3109/07853890.2010.518623

26. Nones RB, Ivantes CP, Pedroso MLA (2017) Can FIB4 and NAFLD fibrosis scores help endocrinologists refer patients with non-alcoholic fatty liver disease to a hepatologist? Arch Endocrinol Metab 61(3):276-281. https://doi. org/10.1590/2359-3997000000233

27. Cicero AF, D'Addato S, Reggi A, Reggiani GM, Borghi C (2013) Hepatic steatosis index and lipid accumulation product as middle-term predictors of incident metabolic syndrome in a large population sample: data from the Brisighella Heart Study. Intern Emerg Med 8(3):265-267. https://doi.org/10.1 007/s11739-012-0875-9

28. Kim JH, Kwon SY, Lee SW, Lee CH (2011) Validation of fatty liver index and lipid accumulation product for predicting fatty liver in Korean population. Liver Int 31(10):1600-1601. https://doi.org/10.1111/j.1478-3231.2011.02580.x

\section{Publisher's Note}

Springer Nature remains neutral with regard to jurisdictional claims in published maps and institutional affiliations.

\section{Submit your manuscript to a SpringerOpen ${ }^{\circ}$ journal and benefit from:}

- Convenient online submission

- Rigorous peer review

- Open access: articles freely available online

- High visibility within the field

- Retaining the copyright to your article

Submit your next manuscript at $\boldsymbol{\wedge}$ springeropen.com 